\title{
Studies of Radiation Damage in Silicon Photomultipliers for the Fermilab Mu2e Cosmic Ray Veto System
}

\author{
Kurt Francis 12 \\ Northern Illinois University \\ E-mail: kfrancis@nicadd.niu.edu
}

The Mu2e experiment at Fermilab will search for the coherent neutrinoless conversion of a muon to an electron in the field of an atomic nucleus with a sensitivity of four orders of magnitude better than previous experiments. The Muze detector is surrounded by a cosmic ray veto system (CRV) that is required to reduce the cosmic-ray background to 0.10 events over the course of the run with an efficiency of 99.99\%. The CRV consists of four layers of scintillator strips with embedded wavelengthshifting fibers and silicon photomultiplier (SiPM) readout. The SiPMs will be exposed to a neutron background which over time may damage the sensors. Several different model SiPMs were irradiated at a proton beam facility. The dark current, dark rate, single photo-electron resolution, cross-talk and response before and after exposure will be presented. A comparison of an equivalent neutron irradiation will also be given.

38th International Conference on High Energy Physics

3-10 August 2016

Chicago, USA

1 Speaker.

2 On behalf of the Mu2e Collaboration. 


\section{Introduction}

The goal of the Mu2e experiment is to search for the neutrino-less conversion of muonsto-electrons, an example of a charged lepton flavor changing process (CLFV). The experiment will measure the ratio of the rate of coherent conversion of negatively charged muons to electrons in the Coulomb field of a nucleus to the kinematically similar muon capture process at a sensitivity of about $3 \times 10^{-17}$, four orders of magnitude better than previous experiments. The Mu2e detector is surrounded by a cosmic ray veto system (CRV) that is required to reduce the cosmic-ray background to 0.10 events over the course of the run with an efficiency of 99.99\%. The CRV consists of four layers of scintillator strips with embedded wavelengthshifting fibers and silicon photomultiplier (SiPM) readout. The SiPMs will be exposed to a neutron background which over time may damage the sensors. Several different model SiPMs were irradiated at a proton beam facility. The dark current, dark rate, single photo-electron resolution, cross-talk and response before and after exposure will be presented.

\section{CRV SiPM Requirements}

Two SiPM photodetectors are placed at each end of the CRV counter. This is needed: (1) to obtain the required photostatistics, (2) for calibration purposes, and (3) to provide redundancy. The sum of the two photodetectors on each end should provide the photoelectron (PE) yield of $25 \mathrm{PE} / \mathrm{cm}$ from a muon at one meter from the counter end in order to keep the CRV system efficiency at the required level of $99.99 \%$.

The SiPM noise rate at the largest expected dose of $1 \times 10^{10} 1 \mathrm{MeV} \mathrm{n} / \mathrm{cm}^{2}$ must allow single photoelectrons to be identified in order that a reliable energy threshold can be set. The hit rate from neutrons/gammas in each SiPM should be no more than $1 \mathrm{MHz}$. The SiPM photoelectron threshold from the above requirement must be less than the threshold needed to meet the overall CRV efficiency requirement. The SiPM and associated front-end electronics must produce a timing resolution no worse than $5 \mathrm{~ns}$ at $90 \% \mathrm{CL}$. The SiPM must be able to meet the above requirements after a total radiation dose of $1 \times 10^{10}$ (1 MeV equivalent) $\mathrm{n} / \mathrm{cm}^{2}$ over the course of the experiment.

\section{SiPM testing}

The critical photodetector parameters (the single PE spectrum, the response to LED pulse, the dark current rate and the cross talk) are measured for a non-irradiated sets of devices of a given type.

The same sets of sensors are exposed to different doses at the proton therapy facility (200 MeV beam) in Warrenville, IL. The measurements were repeated after irradiation and after a short annealing period, and then compared with the original results.

The standard measurements performed on the sensors are: 1) a plot of the current versus voltage applied to the device (IV-curve). From this measurement, the breakdown voltage can be determined and the bias current through the range of working voltages. 2) dark current measurements in which the device has a bias applied to it, at several different voltages including $2.5 \mathrm{~V}$ over breakdown but with no light is applied. From these measurements the noise rate can be determined. Also, thermal noise can produce signals of one and two PE. 3) A low level of LED illumination. These have enough light to generate one to four PE signals. the separation of the peaks in the PE spectrum with give a gain of the device in terms of ADC 
counts/PE. 4) a high level of LED illumination that allows for a determination of the response level before and after irradiation.

We have tested the $1.0 \mathrm{~mm} \times 1.0 \mathrm{~mm}$ and $1.3 \times 1.3 \mathrm{~mm}$ sensors, and have started tests of the $2.0 \mathrm{~mm} \times 2.0 \mathrm{~mm}$ devices

\section{Primary Radiation Damage Effects}

After irradiation the following effects are observed: 1) The IV-curves show a much higher bias current and the breakdown voltage is lower. Figure 1 compares the Bias current before and after irradiation. Irradiation by neutrons with a dose of $6.8 \times 10^{10} \mathrm{p} / \mathrm{cm}^{2}$ were also performed and the effects were proportional to the proton doses consistent with expectations. 2) The dark current noise rate is higher. Table 1 contains the noise rate measurements before and after irradiation. 3) When using the low level LED data, the separate PE peaks are less clearly separated and at the highest irradiation, are impossible to separate and therefore make it impossible to measure the gain, see Figure 2.

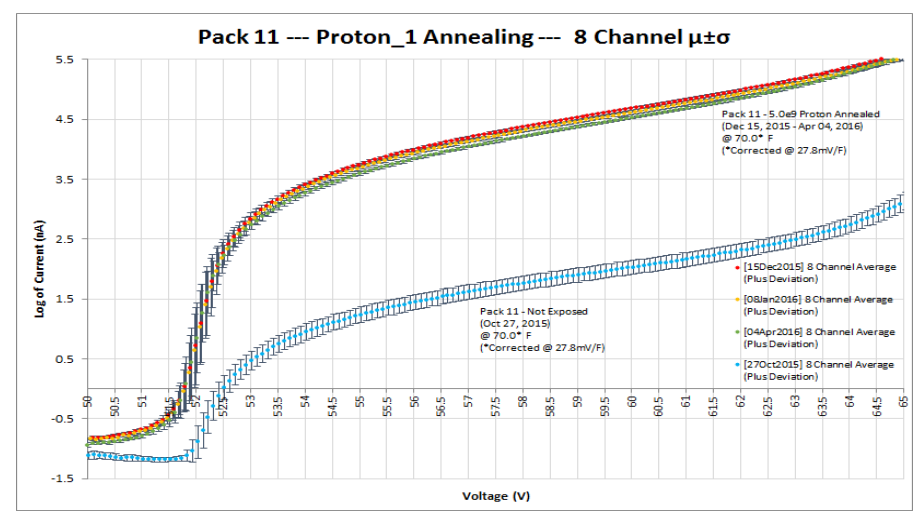

Figure 1. Dark Current/Voltage response before irradiation and after irradiation and annealing for protons $5 \times 10^{9} \mathrm{p} / \mathrm{cm}^{2}$.

Table 1: Noise Rates in 1.3mm x 1.3mm, S13360-1350 SiPMs.

\begin{tabular}{|l|l|l|}
\hline \multicolumn{3}{|c|}{$1.3 \mathrm{~mm} \times 1.3 \mathrm{~mm}, \mathrm{~S} 13360-1350$} \\
\hline V_bias, V & $52.4(0.6$ V_over $)$ & $53.3\left(1.5 \mathrm{~V} \_\right.$over $)$ \\
\hline Un-irradiated, DCR@3.0 PE, KHz & $13-25$ & $25-40$ \\
\hline Un-irradiated, DCR@3.5 PE, KHz & 0.0 & 0.0 \\
\hline $5 \times 10^{9} \mathrm{p} / \mathrm{cm}^{2}$, DCR @3.0 PE, KHz & $0.6-1.6$ & $11-25$ \\
\hline $5 \times 10^{9} \mathrm{p} / \mathrm{cm}^{2}$, DCR@3.5 PE, KHz & $0.0-0.1$ & $2-5$ \\
\hline $1 \times 10^{10} \mathrm{p} / \mathrm{cm}^{2}$, DCR@3.0 PE, KHz & $3-10$ & $22-96$ \\
\hline $1 \times 10^{10} \mathrm{p} / \mathrm{cm}^{2}$, DCR@3.5 PE, KHz & $0.3-1.7$ & $4-25$ \\
\hline
\end{tabular}




\section{Other Effects}

The crosstalk between SiPM pixels after irradiation is also increases. For example, for the $1.3 \mathrm{~mm} \times 1.3 \mathrm{~mm}$ device (S13360-1350) at a bias of 1.5V over breakdown the crosstalk increase from $0.2-2.6 \%$ when un-irradiated to $12.5-19.3 \%$ after irradiation. We also observe a gain reduction (calculated as the relative change in the distance between peaks of one and two PE in photoelectron spectrums). For example, the gain is reduced by approximately $4 \%$ with the $\$ 13360-1350$ after irradiation by $5 \times 10^{9} \mathrm{p} / \mathrm{cm}^{2}$.
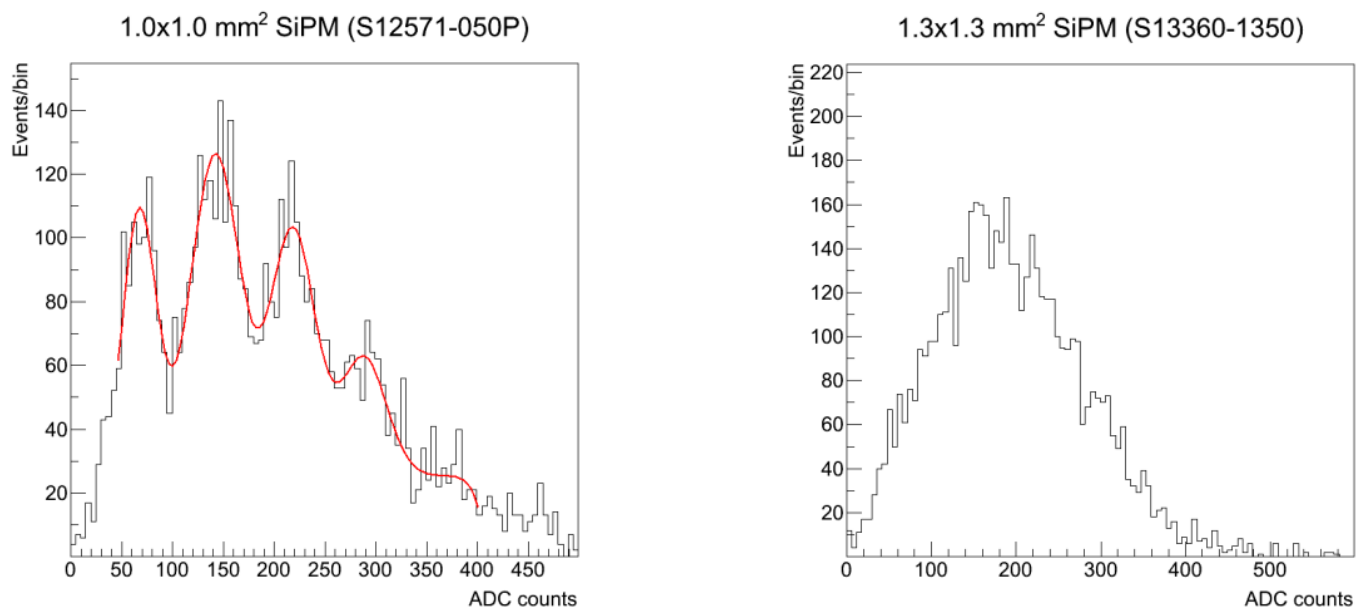

Figure 2. The PE spectrums from $1.0 \times 1.0 \mathrm{~mm} 2$ (left) and $1.3 \times 1.3 \mathrm{~mm} 2$ (right) SiPM after exposure to $5 \times 1010$ protons/cm2 flux. For the $1.3 \times 1.3 \mathrm{~mm} 2$ sensors the $P E$ peaks are no longer visible.

\section{Summary}

Radiation damage measurements were performed on $1.0 \mathrm{~mm} \times 1.0 \mathrm{~mm}$ and $1.3 \mathrm{~mm} \times$ $1.3 \mathrm{~mm}$ devices. The results shown have already given us a very good idea of what to expect. Data from $2 \mathrm{~mm} \times 2 \mathrm{~mm}$ devices has been collected and is being analyzed.

\section{Acknowledgements}

We are grateful for the vital contributions of the Fermilab staff and the technical staff of the participating institutions. We wish to thank people of the proton therapy center in Warrenville, IL for generous use of their facility. This work was supported by the US Department of Energy; the Italian Istituto Nazionale di Fisica Nucleare; the US National Science Foundation; the Ministry of Education and Science of the Russian Federation; the Thousand Talents Plan of the Republic of China; the Helmholtz Association of Germany; and the EU Horizon 2020 Research and Innovation Program under the Marie Sklodowska-Curie Grant Agreement No.690385. Fermilab is operated by Fermi Research Alliance, LLC under Contract No. De-AC02-07CH11359 with the US Department of Energy.

\section{References}

[1] L. Bartoszek et. al. “Mu2e Technical Design Report”, arXiv:1501.05241 (2015). 